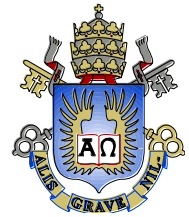

Eric Cardona Romani

Fabricação e caracterização óptica e morfológica de nanopartículas de ouro em substrato vítreo

Dissertação de Mestrado

Dissertação apresentada como requisito parcial para obtenção do título de Mestre pelo Programa de Pós-Graduação em Física da PUC-Rio.

Orientadora: Profa. Isabel Cristina dos Santos Carvalho Co-orientadora: Profa ${ }^{a}$ Paula Medeiros Proença de Gouvêa 
Eric Cardona Romani

\section{Fabricação e caracterização óptica e morfológica de nanopartículas de ouro em substrato vítreo}

Dissertação apresentada como requisito parcial para obtenção do título de Mestre pelo Programa de PósGraduação em Física da PUC-Rio. Aprovada pela Comissão Examinadora abaixo assinada.

Prof ${ }^{a}$. Isabel Cristina dos Santos Carvalho Orientadora Departamento de Física - PUC-Rio

Profa. Paula Medeiros Proença de Gouvêa Co-orientadora Departamento de Engenharia Mecânica - PUC-Rio

Prof $^{\circ}$.Sidnei Paciornik Departamento de Engenharia de Materiais - PUC-Rio

Prof $^{\circ}$. Cristiano Cordeiro UNICAMP

Prof. José Eugênio Leal Coordenador Setorial do Centro

Técnico Científico, PUC - Rio 
Todos os direitos reservados. É proibida a reprodução total ou parcial do trabalho sem autorização da universidade, do autor e do orientador.

Eric Cardona Romani

Graduou-se em Bacharelado em Matemática Aplicada pela Pontifícia Universidade Católica do Rio de Janeiro (PUC) em 2008.

Ficha Catalográfica

Romani, Eric Cardona

Fabricação e caracterização óptica e morfológica de nanopartículas de ouro em substrato vítreo / Eric Cardona Romani; orientadoras: Isabel Cristina dos Santos Carvalho, Paula Medeiros Proença de Gouvêa. - 2011.

122 f. : il.(color.) ; $30 \mathrm{~cm}$

Dissertação

(mestrado)-Pontifícia Universidade Católica do Rio de Janeiro, Departamento de Física, 2011.

Inclui bibliografia 1. Física - Teses. 2. Ressonância de plasmon de superfície localizado. 3. Optica não-linear. 4. Nanopartículas de ouro. I. Carvalho, Isabel Cristina dos Santos. II. Gouvêa, Paula Medeiros Proença de. III. Pontifícia Universidade Católica do Rio de Janeiro. Departamento de Física. IV. Título. 
Aos meus familiares, esposa e aos amigos que vem acompanhando minha jornada. 


\section{Agradecimentos}

Às minhas orientadoras Prof ${ }^{a}$. Dr ${ }^{\mathrm{a}}$. Isabel Carvalho e Dr ${ }^{\mathrm{a}}$. Paula Gouvêa a qual venho trabalhando desde 2007, sendo privilegiado pela excelente orientação, e pela disponibilidade com que tiveram em esclarecer indagações relevantes a pesquisa.

Ao Prof. Dr. Rodrigo Prioli e Dr. Sidnei Paciornik pelas discussões constantes com respeito à morfologia das Nps como também pelo suporte técnico prestado a pesquisa.

À CNPq, à Rede Nanofóton e à PUC-Rio pelos auxílios concedidos, sem os quais este trabalho não poderia ter sido realizado.

À minha família, por todo apoio, paciência e compreensão que tiveram ao longo do Mestrado.

A minha amiga Paula Galvão Caldas pelas inquietantes discussões e esclarecimentos com respeito à ciência e a realidade, pelos trabalhos publicados em anais de congressos e dos seminários PIBIC.

Aos meus colegas Rafael Mendes dos Santos, Rafael Coutinho, André Tavares, Mary, Fernando Fávero, Alexandre Câmara e Hélio Ricardo.

Ao pessoal administrativo e corpo docente do Departamento de Física, ao Departamento de Ciências dos Materiais em especial ao corpo técnico do Laboratório de Microscopia Eletrônica. 


\section{Resumo}

Romani, Eric de Cardona; Carvalho, Isabel Cristina dos Santos; Gouvêa, Paula Medeiros Proença. Fabricação e caracterização óptica e morfológica de nanopartículas de ouro em substrato vítreo. Rio de Janeiro, 2011. 122p. Dissertação de Mestrado - Departamento de Física, Pontifícia Universidade Católica do Rio de Janeiro.

Nesta dissertação descrevemos a preparação de nanopartículas (NPs) de ouro através do aquecimento de filmes finos de ouro depositados em substratos de sodalime pela técnica Magnetron Sputtering. Foi realizado um estudo para cinco espessuras diferentes variando entre (6-20) nm. Descrevemos também a caracterização morfológica e óptica (linear e não-linear) das nanopartículas formadas. Os resultados indicaram que a temperatura é de fundamental importância na formação das NPs, tendo sido realizado um estudo para formação a diferentes temperaturas. As amostras foram caracterizadas estruturalmente pela microscopia de força atômica (AFM) e microscopia eletrônica de varredura de emissão de campo (FESEM). Para o processamento das imagens geradas pelas microscopias utilizou-se uma análise de imagem (IA) antes da extração dos atributos morfológicos envolvendo as etapas de pré-processamento, segmentação, pós-processamento e medida. Para o processamento e extração de atributos, utilizou-se o Software KS400. As partículas possuem dimensões nanométricas com forma aproximadamente esféricas e diâmetros médios em torno de $13 \mathrm{~nm}$ para NPs formadas pelo aquecimento de filmes finos de $6 \mathrm{~nm}$ de espessura à $600^{\circ} \mathrm{C}$ por 4 minutos. As caracterizações ópticas lineares e não-lineares das NPs em estudo foram realizadas através das técnicas de espectroscopia UV-Vis e varredura z (Z-SCAN) em $800 \mathrm{~nm}$ respectivamente. No estudo da não-linearidade óptica foram determinados os índices de refração não linear $\left(\mathrm{n}_{2}\right)$ e a absorção nãolinear $\left(\alpha_{2}\right)$ das NPs de ouro. Os resultados revelaram altos índices de refração nãolinear, assim como uma inversão de sinal na absorção não-linear e no índice de refração não-linear quando os filmes finos de ouro são submetidos a um aquecimento a $600^{\circ} \mathrm{C}$ por 4 minutos.

\section{Palavras-chave}

Ressonância de Plasmon de Superfície Localizado; óptica não-linear; nanopartículas de ouro. 


\section{Abstract}

Romani, Eric de Cardona; Carvalho, Isabel Cristina dos Santos (Advisor); Gouvêa, Paula Medeiros Proença (Co-Advisor). Fabrication and optical and morphological characterization of gold nanoparticles on glass substrate. Rio de Janeiro, 2011. 122p. MSc Dissertation - Departamento de Física, Pontifícia Universidade Católica do Rio de Janeiro.

In this dissertation we describe the preparation of gold nanoparticles (NPs) by heating gold thin films of thicknesses between (6-20) nm, previously deposited on soda-lime substrates by Magnetron Sputtering. Also described are the morphological and optical (linear and nonlinear) characterization of the NPs. The tempertature dependence of the formation process of the NPs was investigated. The samples were structurally characterized by Atomic Force Microscopy (AFM) and Scanning Electron Microscopy, High Resolution (FESEM). We used an image analysis (IA) before extraction of morphological features involving the steps of preprocessing, segmentation, post-processing and measurement. For the processing and extraction of attributes, we used the software KS400. The particles have nanometers dimensions with approximately spherical shape and diameters around $13 \mathrm{~nm}$ for NPs formed by heating $6 \mathrm{~nm}$ thick thin films at $600{ }^{\circ} \mathrm{C}$ for 4 minutes. The linear and nonlinear optical characterization of the NPs were performed through the techniques of UV-Vis spectroscopy and (TM-EZ at 532 $\mathrm{nm}$, respectively. The non-linear optical characterization yielded the nonlinear refractive index (n2) and nonlinear absorption $\left(\alpha_{2}\right)$ of the gold NPs formed. The results revealed high linear refractive indices, as well as a sign inversion of the nonlinear absorption when the NPs were formed.

\section{Keywords}

Localized Surface Plasmon Resonance; non-linear optics; gold nanoparticles. 


\section{Sumário}

1 Introdução

2 Nanopartículas metálicas 20

2.1. Introdução 20

2.2. Propriedades eletrônicas 22

2.3 Propriedades ópticas 23

2.3.1 Coeficiente de extinção na Teoria de Mie 23

2.3.2 Campo dipolar e aproximação quase-estática 25

2.3.3 Modelo de Maxwell-Garnett para nanopartículas metálicas 33

2.3.4 Ressonância de Plasmon de Superfície localizado 41

2.3.4.1 Deslocamento da frequência de ressonância em NPs metálicas 43

2.3.5 Resultados de simulação e conclusão 45

3 Óptica Não-linear 49

3.1. Não-linearidade de terceira ordem 50

3.2. Técnica Z-scan 54

4 Fabricação e Caracterização de Nanopartículas de Ouro 58

4.1. Fabricação de Nanopartículas de Ouro 60

4.2 Caracterização morfológica e Processamento de imagens (IA) 63

4.2.1 Microscopia de Força Atômica 64

4.2.2 Microscopia Eletrônica de Varredura de Emissão de Campo (FESEM)

4.2.3 Processamento de imagens no KS400 74

4.3 Caracterização Óptica linear 88

4.4 Caracterização Óptica Não-linear 94

5 Códigos e cálculos complementares 99

5.1 Aproximação dipolar quase estática 99

5.2 Parte real e imaginária do índice de refração 109 
5.3 Código do KS400

5.4 Código de simulação do Maple 14

6 Conclusão

7 Bibliografia 


\section{Lista de figuras}

Figura 1: Esquema representando os níveis de energia eletrônicos em materiais metálicos. $\mathrm{O}$ aumento do número de átomos provoca a transição do discreto para o contínuo. Em cinza, estados eletrônicos desocupados ${ }^{18}$.

Figura 2: Esquema retratando a diferença da relação raio da partícula metálica com comprimento da luz incidente. (a) um problema mais complicado envolvendo um campo não uniforme em torno da partícula metálica, enquanto que em (b) temos o caso mais simples em que o raio da partícula é muito menor em relação ao comprimento de onda da luz incidente. Neste caso o campo pode ser considerado como sendo constante em torno da partícula metálica.

Figura 3: Esfera homogênea e isotrópica imersa em um campo elétrico $E_{o}$ estático na direção $\mathrm{z}$.

Figura 4: Gráficos da parte real $\left(\varepsilon_{1}\right)$ e imaginária $\left(\varepsilon_{2}\right)$ da constante dielétrica de Lorentz para osciladores de elétrons ${ }^{26}$.

Figura 5: Gráficos da parte real $\left(\varepsilon_{1}{ }^{D}\right)$ e imaginária $\left(\varepsilon_{2}{ }^{D}\right)$ da constante dielétrica de Drude para materiais com elétrons livres ${ }^{26}$.

Figura 6: Imagem ilustrativa do efeito LSPR (Ressonância de Plasmon de Superfície Localizado).

Figura 7: Gráfico da parte real da constante dielétrica de Maxwell-Garnett utilizando-se a constante dielétrica de Drude para o ouro, no caso em que $\varepsilon_{m}=1$ e f $=0,02$.

Figura 8: Gráfico da parte imaginária da constante dielétrica de Maxwell-Garnett utilizando-se a constante dielétrica de Drude para o ouro, no caso em que $\varepsilon_{m}=1$ e f $=0,02$.

Figura 9: Gráfico da parte real da constante dielétrica de Maxwell-Garnett utilizando-se a constante dielétrica de Drude para o ouro, no caso em que $\varepsilon_{m}=1$ e varia (f $\left.=0,02 ; 0,04 ; 0,06 ; 0,08 ; 0,1\right)$.

Figura 10: Gráfico da parte imaginária da constante dielétrica de Maxwell-Garnett se utilizando da constante dielétrica de Drude para o 
ouro no caso em que $\varepsilon_{m}=1$ e varia (f= 0,$\left.02 ; 0,04 ; 0,06 ; 0,08 ; 0,1\right)$.

Figura 11: absorbância das NPs em função do comprimento de onda para 3 fatores de preenchimento $(f=0,02 ; f=0,03 ; f=0,04)$.

Figura 12: Esquema ilustrativo do experimento proposto por Sheik Bahae para medir o índice de refração não-linear e a absorção não-linear.

Figura 13: Esquema ilustrativo mostrando a obtenção da transmitância em função da posição de varredura $Z^{33}$.

Figura 14: Gráfico da transmitância normalizada em função da varredura $\mathrm{Z}$ do filme de ouro antes do aquecimento a $600^{\circ} \mathrm{C}$ por 4 minutos.

Figura 15: Gráfico da transmitância normalizada em função da varredura $\mathrm{Z}$ do filme de ouro depois do aquecimento a $600^{\circ} \mathrm{C}$ por 4 minutos.

Figura 16: soda-lime da Roni-Alzi (26 x 76 mm) lapidadas.

Figura 17: Gráfico de calibração para determinação do tempo de deposição.

Figura 18: Filme fino de ouro de $6 \mathrm{~nm}$ de espessura antes do aquecimento

(a) e depois do aquecimento (b).

Figura 19: Microscópio de Força Atômico do laboratório de Nanoscopia do Departamento de Física coordenado pelo professor Rodrigo Prioli.

Figura 20: Representação ilustrativa do fenômeno "convolução" que ocorre quando o raio da ponta é da ordem do grão que esta sendo analisado.

Figura 21: Imagens obtidas pelo AFM do filme de ouro antes (a) e depois

(b) do aquecimento a $600^{\circ} \mathrm{C}$ por 4 minutos para a região de $2 \mu \mathrm{m}$ x $2 \mu \mathrm{m}$.

Figura 22: Imagens obtidas pelo AFM do filme de ouro antes (a) e depois

(b) do aquecimento a $600^{\circ} \mathrm{C}$ por 4 minutos para a região de $1 \mu \mathrm{m} \times 1 \mu \mathrm{m}$.

Figura 23: Imagem gerada pelo AFM da amostras de filme de Au de $6 \mathrm{~nm}$ de espessura sobre substrato de vítreo antes do tratamento térmico (a). Perfil longitudinal (b) e transversal (c) da altura das NPs da imagem (a).

Figura 24: Imagem gerada pelo AFM da amostras de filme de Au de 6nm de espessura sobre substrato de vítreo após o tratamento térmico (a). Perfil longitudinal (b) e transversal (c) da altura das NPs da imagem (a). Figura 25: Conjunto de imagens geradas pelo AFM para as 5 espessuras de filme de ouro diferentes antes e depois do aquecimento à $600^{\circ} \mathrm{C}$ por $4 \mathrm{~min}$. Figura 26: Microscópio Eletrônico de Varredura de Emissão de Campo do Laboratório de Microscopia do Dept. de Física da PUC-Rio. 
Figura 27: Imagem gerada pelo FESEM da amostra de 6nm de espessura depois do aquecimento em um aumento de 100.000x a uma distância de trabalho de $8,2 \mathrm{~mm}$.

Figura 28: Conjunto de imagens geradas pelo FESEM das amostras de vidro de soda-lime com filme de ouro ( $8 \mathrm{~nm}, 10 \mathrm{~nm}, 12 \mathrm{~nm}$ e $20 \mathrm{~nm})$ depositado, antes e depois do aquecimento a $600^{\circ} \mathrm{C}$ por 4 minutos.

Figura 29: Etapas do processamento digital da imagem gerada pelo AFM.

Imagem sem processamento e evidenciando uma iluminação não homogênea (a), imagem pré-processada utilizando os filtros (Plane, Equalize e Flatten) do Wsxm (b), imagem em tons de cinza (c), imagem após a aplicação da limiarização e detecção de bordas com o algoritmo de Canny (d), imagem obtida pela aplicação do divisor de águas (WaterSheds) (e) e a imagem com a máscara final (f).

Figura 30: Imagem original (a), máscara sobre a imagem original em tons de cinza (b).

Figura 31: Histograma da distribuição da altura (MAXD) das NPs fabricadas pelo aquecimento do filme fino de ouro de $6 \mathrm{~nm}$ de espessura a $600^{\circ} \mathrm{C}$ por 4 minutos.

Figura 32: Etapas do processamento da imagem gerada pelo AFM do filme fino de ouro de $6 \mathrm{~nm}$ de espessura antes do aquecimento.

Figura 33: Histograma da distribuição da altura das NPs obtida pelo KS400 do filme fino de ouro de $6 \mathrm{~nm}$ de espessura antes do aquecimento.

Figura 34: Histograma da distribuição da altura obtido pelo KS400 das Nps geradas pelo aquecimento do filme fino de ouro de $6 \mathrm{~nm}$ de espessura. 80 Figura 35: Etapas do processamento da imagem gerada pelo FESEM da amostra com NPs fabricadas pelo aquecimento do filme fino de $6 \mathrm{~nm}$ de espessura a $600^{\circ} \mathrm{C}$ por 4 minutos.

Figura 36: Máscaras obtidos pelo KS400 das imagens do AFM (a) e FESEM (b) da amostra com NPs de ouro fabricadas pelo aquecimento do filme fino de $6 \mathrm{~nm}$ de espessura à $600^{\circ} \mathrm{C}$ por 4 minutos.

Figura 37: Imagem obtida pelo FESEM da amostra com NPs fabricadas pelo aquecimento do filme fino de ouro de $12 \mathrm{~nm}$ de espessura.

Figura 38: Imagem obtida pelo FESEM da amostra com NPs fabricadas 
pelo aquecimento do filme fino de ouro de $20 \mathrm{~nm}$ de espessura.

Figura 39: Geometria das grandezas extraídas das imagens geradas pelas microscopias.

Figura 40: Histogramas: Diâmetro das NPs (a), fator de forma circular (b) e razão de aspectos (c) obtidos pelo KS400 das imagens geradas pelo FESEM da amostra de $6 \mathrm{~nm}$ de espessura aquecida a $600^{\circ} \mathrm{C}$ por 4 minutos.

Figura 41: Absorbância da amostra de filme de Au de $6 \mathrm{~nm}$ de espessura sobre o substrato de soda-lime antes e depois do aquecimento a $600^{\circ} \mathrm{C}$ por 4 minutos.

Figura 42: Absorbância da amostra de filme de Au de $8 \mathrm{~nm}$ de espessura sobre o substrato de soda-lime antes e depois do aquecimento a $600^{\circ} \mathrm{C}$ por 4 minutos.

Figura 43: Absorbância da amostra de filme de Au de $10 \mathrm{~nm}$ de espessura sobre o substrato de soda-lime antes e depois do aquecimento a $600^{\circ} \mathrm{C}$ por 4 minutos.

Figura 44: Absorbância da amostra de filme de Au de $12 \mathrm{~nm}$ de espessura sobre o substrato de soda-lime antes e depois do aquecimento a $600^{\circ} \mathrm{C}$ por 4 minutos.

Figura 45: Absorbância da amostra de filme de Au de $20 \mathrm{~nm}$ de espessura sobre o substrato de soda-lime antes e depois do aquecimento a $600^{\circ} \mathrm{C}$ por 4 minutos. (a) e absorção do filme de ouro volumétrico ${ }^{45}$.

Figura 46: Espectro de absorção antes do aquecimento a $600^{\circ} \mathrm{C}$ por 4 minutos das amostras com cinco espessuras diferentes (6 nm, $8 \mathrm{~nm}, 10 \mathrm{~nm}, 12 \mathrm{~nm}$ e 20nm) sobre o substrato de soda-lime.

Figura 47: Espectro de absorção depois do aquecimento a $600^{\circ} \mathrm{C}$ por 4 minutos das amostras com cinco espessuras diferentes (6 nm, $8 \mathrm{~nm}, 10 \mathrm{~nm}, 12 \mathrm{~nm}$ e $20 \mathrm{~nm}$ ) sobre o substrato de soda-lime. (a). Comportamento da variação do diâmetro das NPs em função da espessura (b).

Figura 48: Absorbância da amostra de filme de Au de 6 nm de espessura sobre o substrato de soda-lime aquecida a temperaturas diferentes $\left(200^{\circ} \mathrm{C}, 400^{\circ} \mathrm{C}, 600^{\circ} \mathrm{C}\right.$ e $\left.800^{\circ} \mathrm{C}\right)$.

Figura 49: Espectro de absorção depois do aquecimento a $270^{\circ} \mathrm{C}$ 
por 4 minutos para 3 espessuras $(6 \mathrm{~nm}, 8 \mathrm{~nm}$ e $10 \mathrm{~nm})$ sobre o substrato de soda-lime .

Figura 50: Espectro de absorção depois do aquecimento a $410^{\circ} \mathrm{C}$ por 4 minutos para 3 espessuras $(6 \mathrm{~nm}, 8 \mathrm{~nm}$ e $10 \mathrm{~nm})$ sobre o substrato de soda-lime .

Figura 51: Espectro de absorção depois do aquecimento a $600^{\circ} \mathrm{C}$

por 4 minutos para 3 espessuras $(6 \mathrm{~nm}, 8 \mathrm{~nm}$ e $10 \mathrm{~nm})$ sobre o substrato de soda-lime .

Figura 52: Espectro de absorção depois do aquecimento a $800^{\circ} \mathrm{C}$ por 4 minutos para 3 espessuras $(6 \mathrm{~nm}, 8 \mathrm{~nm}$ e $10 \mathrm{~nm})$ sobre o substrato de soda-lime .

Figura 53: (a) Montagem experimental do TM-EZ scan. (b)

Transmitância normalizada em função do tempo de abertura do chopper para a amostra de $\mathrm{CS}_{2}$.

Figura 54: Evolução temporal do sinal normalizado do TM-EZ scan para as posições pré-focal (curvas crescentes) e pós-focal (curvas decrescentes) da amostra referente a amostra com filme de Au de espessura de $6 \mathrm{~nm}$ (curva preta) e após tratamento térmico a $600^{\circ} \mathrm{C}$ e 4 minutos (curva vermelha).

Figura 55: Curvas normalizadas de transmissão para a amostra com filme de Au de espessura de 6nm antes (círculo aberto) e após (quadrado aberto) tratamento térmico a $600^{\circ} \mathrm{C}$ e 4 minutos. 


\section{Lista de tabelas}

Tabela 1: Resumo das condições de contorno para o campo elétrico E.

Do lado esquerdo encontram-se as condições associadas às partes interna e externa à esfera metálica (nanopartícula), enquanto que do lado direito as condições associadas à superfície da esfera $(r=R)$.

Tabela 2: Resumo das condições de contorno para o potencial. Do lado esquerdo encontram-se as condições associadas às partes interna e externa à esfera metálica (nanopartícula) enquanto que do lado direito encontram-se as condições associadas à superfície da esfera $(r=R)$.

Tabela 3: Dados estatísticos das imagens processadas pelo KS400 das imagens do AFM das amostras de 6nm, 8nm, 10nm, $12 \mathrm{~nm}$ e $20 \mathrm{~nm}$ aquecidas à $600^{\circ} \mathrm{C}$ por 4 minutos.

Tabela 4: Dados estatísticos das imagens processadas pelo KS400 das imagens do FESEM das amostras de 6nm, 8nm, 10nm, 12nm e $20 \mathrm{~nm}$ aquecidas à $600^{\circ} \mathrm{C}$ por 4 minutos.

Tabela 5: Valores de $\mathrm{n}_{2}$ e $\alpha_{2}$ para 3 diferentes espessuras de filmes de $\mathrm{Au}(6 \mathrm{~nm}, 10 \mathrm{~nm}$ and $20 \mathrm{~nm})$ em substratos de soda-lime antes e depois do aquecimento a $600^{\circ} \mathrm{C}$ por 4 minutos. Substratos de soda-lime apresentaram valores de $\mathrm{n}_{2}=3,0 \times 10^{-16} \mathrm{~cm}^{2} / \mathrm{W}$, que esta de acordo com o obtido por Ghalen et al.

Tabela 6: Resumo das condições de contorno para o campo elétrico E.

Do lado esquerdo a condição associada às partes interna e externa à esfera metálica (nanopartícula) enquanto do lado direito as condições associadas à superfície da esfera $(\mathrm{r}=\mathrm{R})$.

Tabela 7: Resumo das condições de contorno para o potencial. Do lado esquerdo a condição associada às partes interna e externa a esfera metálica (nanopartícula) enquanto do lado direito as condições associadas à superfície da esfera $(\mathrm{r}=\mathrm{R})$. 